\title{
Nurturing Communities of Inquiry: A Formative Study of the DojoIBL Platform
}

\author{
Ángel Suárez ${ }^{(\bowtie)}$, Stefaan Ternier, Fleur Prinsen, and Marcus Specht \\ Welten Institute, Open University of the Netherlands, Heerlen, The Netherlands \\ \{angel. suarez, stefaan. ternier, fleur.prinsen, \\ marcus.specht\}@ou.nl
}

\begin{abstract}
This formative study introduces DojolBL, a web-based platform to support collaborative inquiry-based learning processes. By supporting communication and collaboration with new technological affordances, DojoIBL aims at nurturing communities of inquiry. The study elaborates on the theoretical underpinning of DojoIBL, describes its added value and presents a detailed explanation about the functionalities supported. Thereafter, an evaluation about how users perceived DojoIBL has been performed. Besides, the positive acceptance of participants, the results also showed that DojoIBL seems to be a suitable tool to support essential components of communities of inquiry. The study concludes anticipating the integration of role support as future developments of DojoIBL.
\end{abstract}

Keywords: Inquiry-based learning - Community of Inquiry $\cdot$ Collaborative knowledge building $\cdot$ Context-awareness $\cdot$ Informal learning

\section{Introduction}

In recent years, there has been an increasing interest in socio-constructivist learning methods e.g., (mobile) inquiry-based learning (IBL) [1], as well as the technological tools that support them [2]. IBL is often characterized as a collaborative process, in which informal and formal learning activities are socially interconnected. These activities need to be seamlessly supported in order to provide an effective and complete experience to the students. The collaborative inquiry process was aptly defined in the 'Community of Inquiry' approach [3], which emphasizes that creation of knowledge requires social interactions of individuals with different background knowledge.

However, there is still a lack of research on the technological affordances to enhance the IBL process and nurture a community of inquiry. For instance, the power of cloud based services in combination with instant communication or notifications have not been entirely explored in the context of inquiry-based learning. Previous studies conducted in the context of the weSPOT European project [4] ${ }^{1}$, a three-year project in which experience and knowledge about IBL have been acquired, showed that there were issues integrating and using technology in collaborative IBL processes. These issues were related to the lack of adequate technological affordances nurturing

\footnotetext{
${ }^{1}$ http://inquiry.wespot.net/. 
the communities of inquiry as also the educational settings in which inquiry-based learning often is implemented. Certainly, teachers faced difficulties to encourage and to help students explore topics as a community also due to the complex different variations of inquiry-based learning from confirmation inquiry to open inquiries.

In our effort to study an affordable solution that combines the essential elements to support IBL with the added potential of new technological affordances, this research study contributes DojoIBL, a platform that focuses on supporting 'Community of Inquiry' (CoI).

In the first two sections we will elaborate the theoretical underpinnings of DojoIBL; existing IBL solutions and social collaborative tools are discussed, and the rationale to develop DojoIBL is explained. Next, the design principles of the DojoIBL are described. The added value of DojoIBL, as compared to other IBL solutions, is argued in section four. Thereafter, in section five and six, the research design of the study is introduced and the results of a study into DojoIBL user experiences are described. Section seven elaborates on the interpretation and discussion of the results. Finally, the conclusion and the future work of the DojoIBL platform are outlined.

\section{Theoretical Framework}

Inquiry-based learning is defined on the premise that learning is more than memorizing information, rather it is a process of understanding, developing inquiry skills and constructing knowledge sparked by curiosity [5]. Often, inquiry processes incorporate elements of collaboration, which was defined in [6] as the engagement of students in a common endeavor. Collaboration transforms the inquiry activities into processes of co-construction of knowledge around shared understandings or concepts. Collaborative inquiry learning has also been defined in [7] in its Knowledge building approach, as an unpredictable, holistic process of creative development of ideas within a community of learners [5]. Moreover, socio-constructivist learning theories stated that knowledge is materialized when people, with different background knowledge, collaborate to find answers to a problem.

Community of Inquiry. The definitions of collaborative inquiry-based learning, anticipated the concept of community in IBL. [3] coined the term 'Community of Inquiry' (CoI) to refer to a group of individuals (facilitators and students) transacting with the specific purposes of facilitating, constructing, validating understanding and developing capabilities leading to further learning. In other words, the CoI framework is concerned with the nature of knowledge formation in IBL. [8] already defined it as a continuous exploration of a topic of students' interest, where community members (students) engage in social interactions to generate shared understanding. It has been shown in the literature that text-based communications have a considerable potential to facilitate the creation of communities of inquiry (CoI) [9, 10]. As already mostly evident in the definition given in [11], CoI comprises three essential components to any educational transaction: cognitive presence, which is defined as the capability of each participant in the CoI to construct meaning through sustained communication [9], social presence that relates to the ability of students to positioned themselves socially 
and affectively in the CoI [12] and teaching presence, which is characterized as the design, facilitation and direction of cognitive and social processes in order to produce meaningful co-creation of knowledge [13].

[14] emphasized the need to establish a common ground and perform in a community of practice (even broader than CoI) in order to work and learn efficiently. Notifications and awareness in collaborative activities can contribute to achieve this common ground [15]. [15] defined the three following types of collaboration awareness. Social awareness, relates to the presence of others working in parallel and it involves motivational or attitudinal aspects like timing, frequency or intensity. Action awareness copes with the idea that social awareness is not enough. Besides knowing who is around, students must be informed about what is happening. The last type, activity awareness, advices organizational and structural changes that helps students to understand the context of the inquiry activity.

Social Collaboration Supported with Technology. Research has shown that technology can support inquiry-based learning [16-18]. We attribute this to advancements in technology and its capacity to offer new possibilities for scaffolding the inquiry-based learning process. Premised on the theoretical framework of social constructivism, inquiry-based learning supports co-creation of knowledge through social interactions, between students-students and students-facilitators. Co-Lab [18], an online desktop environment offering an integrated approach for collaboration, modeling and inquiry, already addressed this to promote scientific discovery learning. Other developments such as nQuire $[19]^{2}$, a software application to guide personal inquiry learning, or Go-Lab ${ }^{3}$ [20] (through Graasp ${ }^{4}$ ) a project that provides guided experimentation that helps students acquiring inquiry skills, addressed collaboration. However, these platforms have not yet fully exploit emerging technological affordances. More recently, educational platforms like Edmodo ${ }^{5}$ or ClassDojo ${ }^{6}$ have enabled students to connect and to collaborate using cloud-based and social functionalities similar to the affordances of most popular social network platforms. Edmodo, is a social learning community where students, teachers and parents form communities or groups of their interest. It uses the timeline metaphor to display the latest posts in the communities or groups the user is following. The user's contributions are based on the following four types; notes, assignments, quiz or polls, which allow participants to connect around shared ideas. Comparable, ClassDojo is a communication platform that aims at encourage students to learn in a happier way engaging parents on the process. ClassDojo has three visualizations for the classroom; class story, a timeline visualization of the latest contributions, a classroom visualization where all the students are displayed facilitating students' rewarding and messages visualization to easily connect with others. Both initiatives provide resources to increase students' awareness and communication.

\footnotetext{
${ }^{2}$ http://www.nquire.org.uk/home.

${ }^{3}$ http://www.golabz.eu/.

${ }^{4}$ http://graasp.eu/.

5 https://www.edmodo.com/.

6 https://www.classdojo.com/.
} 
Group awareness has been an emerging topic in Computer Supported Collaborative Learning (CSCL) research [21]. Three types of awareness can be extracted from the above research studies; process, social and activity awareness [14, 15, 22]. Each of the studies focuses on helping students to visualize and manipulate social processes in order to understand how the group moves forward. Moreover, regarding communication, it has been proven in literature that text-based communication has a considerable potential to facilitate the creation of communities of inquiry $(\mathrm{CoI})[3,10]$.

To sum up, current platforms $[19,20]$ have sought to support the IBL process. These platforms have yet to fully harness the affordances of educational and social network platforms (e.g. ClassDojo and Edmodo) and emerging technological tools to support social collaboration and to nurture community of inquiries. Hence, based on existing initiatives and studies, this research explores the affordances of emerging technologies in the design of DojoIBL to foster communities of inquires. Essentially, it investigates how DojoIBL can facilitate social interactions and raise students' awareness of collaborative IBL processes.

\section{Research Design}

This research study introduces DojoIBL, a multi-device Learning Content Management System $^{7}$ (LCMS) to scaffold and to support students' collaborative knowledge co-construction process in IBL. Rather than delivering course content material, DojoIBL provides the designers and the facilitators with the tools to structure IBL processes about any meaningful topic from students' curiosity. Therefore, it focuses on the process rather than in the content itself. DojoIBL has been developed following a design-based research approach [23] in which teachers, designers and researchers collaboratively generate feedback feeding the iterative and incremental development process. Results of the weSPOT European project [4], showed that it is important to involve teachers in the early stages of the design and development process; giving us a broader perspective on the flexibility that the platform should have. The weSPOT project experiences and knowledge encouraged our team to develop DojoIBL, following several design principles that will be summarized.

The weSPOT project showed that students can be overwhelmed if the cognitive requirements demanded by our system are too high. Therefore, one of our aims was to reduce extraneous cognitive load, by ensuring that all elements included in DojoIBL add value to the learning experience. Thus, unnecessary information or elements that distract students from learning have been avoided in the interface, and visual representations of the inquiry process have been used to make the system more intuitive. Moreover, research studies on IBL [24, 25] exemplify the need to scaffold the inquiry learning process hence, DojoIBL breaks down the inquiry process into phases [25], and the phases into activities, in order to provide implicit guidance on the inquiry process.

Inquiry based Learning is a collaborative process $[5,7,26]$ where students also learn from their peers by reflecting and building on top of one another's ideas.

\footnotetext{
${ }^{7}$ https://en.wikipedia.org/wiki/Content_management_system accessed on March 2016.
} 
Hence, DojoIBL implements an instant messaging system supporting cognitive presence, social presence and teaching presence [11-13] which contributes to generate a Community of Inquiry [3, 10]. Yet, students per se are not skilled on acting as a community. Consequently, teachers' orchestration [27] and scaffolding remain essential [28], especially at early stages of the inquiry process. In addition to instant messaging, DojoIBL implements a notification system and an inquiry timeline, which facilitates asynchronous collaboration and raise awareness among students [15].

In short, DojoIBL focuses on adding value to the authentic inquiry experiences, providing an intuitive, simple and flexible tool that enables collaborative self-directed learning for students and just in context - time and place - orchestration for teachers (Fig. 1).

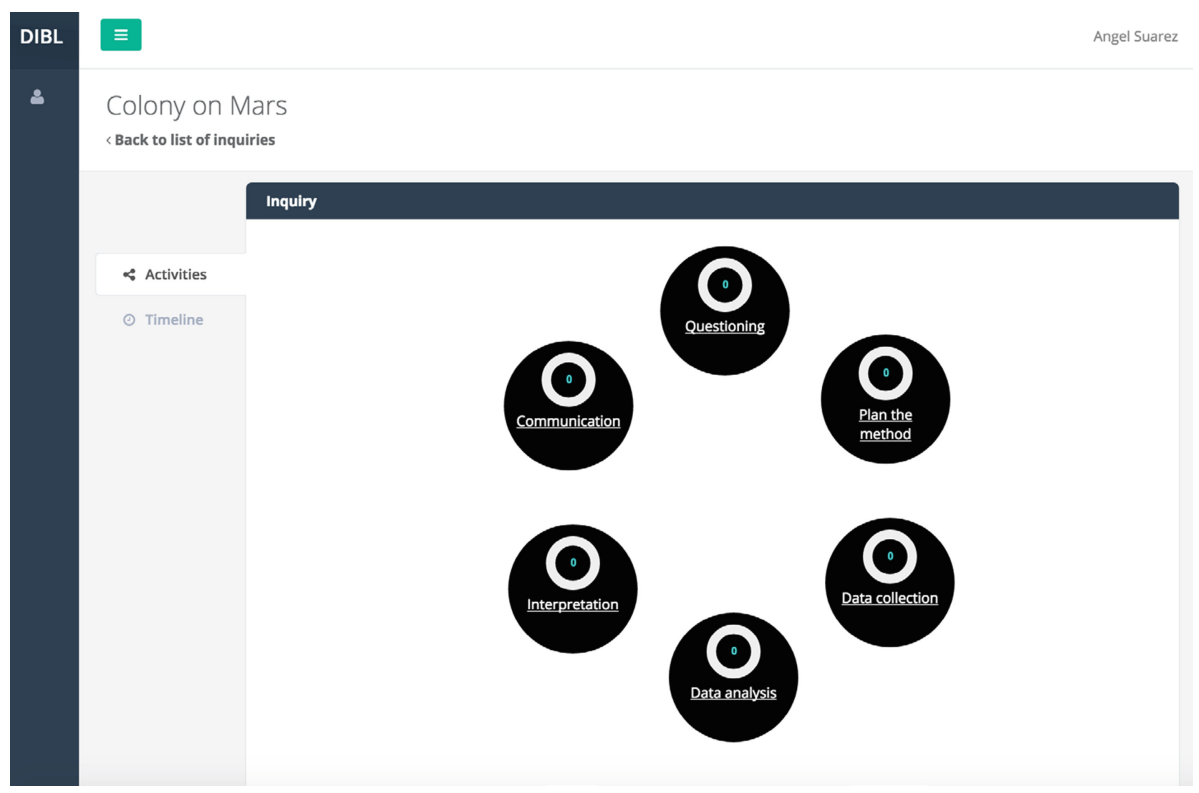

Fig. 1. Visualization of the inquiry process on the Colony on Mars activity

\section{Affordances of DojoIBL}

DojoIBL is an open source platform that builds on the ARLearn framework [29], a PaaS cloud based architecture deployed in Google App Engine (GAE). DojoIBL is a Learning Content Management System that provides atomic inquiry elements to structure collaborative inquiry processes. This section illustrates how the design challenges are addressed in DojoIBL, as well as discusses the added value of DojoIBL as compared to existing IBL solutions.

One of the main characteristic of DojoIBL is that users are able to design blueprints or templates for an inquiry structure. That means, several inquiries can be created based on the same blueprint or template of an inquiry structure. As a consequence, students 
can work in groups on different topics using a common inquiry structure. In addition, similar to what other educational platforms like Spiral.ac ${ }^{8}$ or Edmodo ${ }^{9}$ do, DojoIBL generates unique codes for each inquiry group. Consequently, managing and organizing students in inquiry groups can be reduced to share the specific codes with them. This functionality addresses one of the design requirements introduced before, simplicity.

Another design requirement highlights the necessity to work with intuitive designs and platforms that help students understand the inquiry process. The opportunity to practice, understand and master the steps needed to answer any given question helps students to be more self-directed learners and to be less dependent on facilitators' scaffolding. For instance, existing solutions like nQuire, uses visual representations of the inquiry cycle. In DojoIBL, inspired by those existing solutions, an interactive visualization of the inquiry structure is used (Fig. 1). This visualization builds on the IBL model [4] and represents every inquiry phase as a cycle, that when clicked opens the activities related to this phase.

DojoIBL aims at supporting authentic and transformative [30] inquiry learning processes. Rather than teachers providing the conceptual knowledge, IBL relies on teachers orchestrating and scaffolding the process using different strategies or structures [30]. To help students achieve higher order thinking and to create opportunities for students to develop their inquiry skills and their own understanding around questions, DojoIBL uses atomic inquiry elements. An atomic inquiry element is defined as the smallest re-usable type of activity that can be added to an inquiry phase. Currently, there are six types of activities available in DojoIBL, and each type provides a specific pedagogical affordance:

- The research question is an essential part of IBL where students collaboratively work around a shared question or topic. It aims at developing critical thinking skills $[9,11,31]$, and it must be supported with tools to generate individual discussions, which enables self-directed learning as each student can create his/her own question, and other can contribute to it.

- Discussion forms the simplest type of activity which is based on plain text. Students can find a description, a story or a definition that inspire them about the specific topic. Activities are flexibly enabling any kind of activity design. For example, activities inform the student about the criteria (i.e. rubrics) that the teacher will use to evaluate in that particular activity. This will help students to work towards a save direction (Fig. 2).

- Data collection enables the visualization and uploading of data to DojoIBL. Every piece of research contains some sort of data collection, which very often consist of collecting existing information on the internet or in their environment.

- Concept mapping helps students to represent and organize knowledge and concepts around a topic [32, 33]. We have developed a type of activity that stores the

\footnotetext{
${ }^{8}$ https://spiral.ac/student.

9 https://www.edmodo.com/.
} 


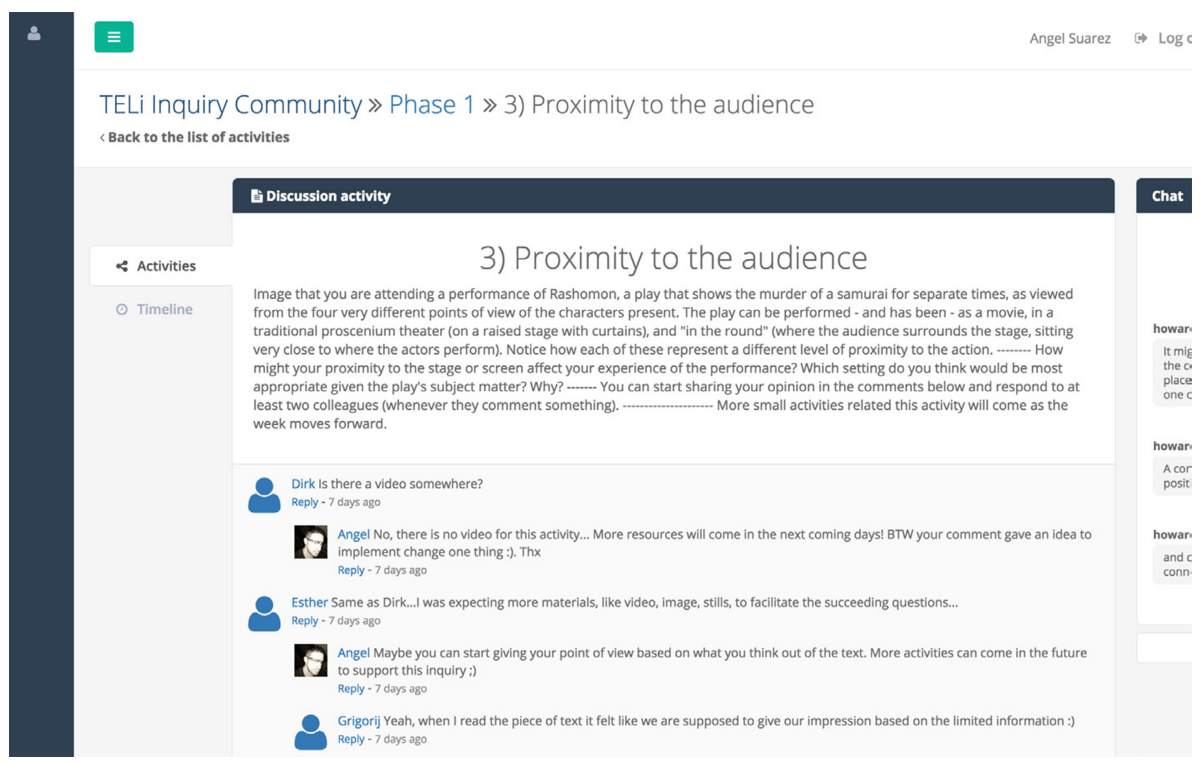

Fig. 2. Example of activity type: discussion.

information on the server, rather than relying on services like Mindmeister ${ }^{10}$ that stores the concept map data externally.

- External plugin enables the integration of external widgets repositories like GoLabs [20]. Those widgets provide the possibility to conduct scientific experiments in a virtual environment.

- Multimedia are similar to discussion activity but it adds the possibility to incorporate a multimedia element to inspire students. The multimedia can be used to support the description of the activity.

The activities are provided with an individual section for comments or explanations. Students can, for example, share, negotiate or compare their ideas. Actually, they can experience what the study [34] defined as the five phases of negotiation and knowledge co-construction: sharing and comparing, dissonance, negotiation, co-construction, testing and application. In addition, in order not to increase extraneous cognitive load for students, the design is inspired on existing social network platforms. The idea is to help students to get confidence with system quickly to speed up the adaptation phase.

The last requirement in the design section was the support of collaboration. The instant messaging system (right side of Fig. 3) offers a communication channel that is contextualized to the inquiry topic, therefore discussions through the chat system are embedded in a context which helps to focus the discussions. The instant messaging facilitates the support of the three essential components of any educational transaction;

\footnotetext{
${ }^{10}$ https://www.mindmeister.com/ accessed on March 2016.
} 


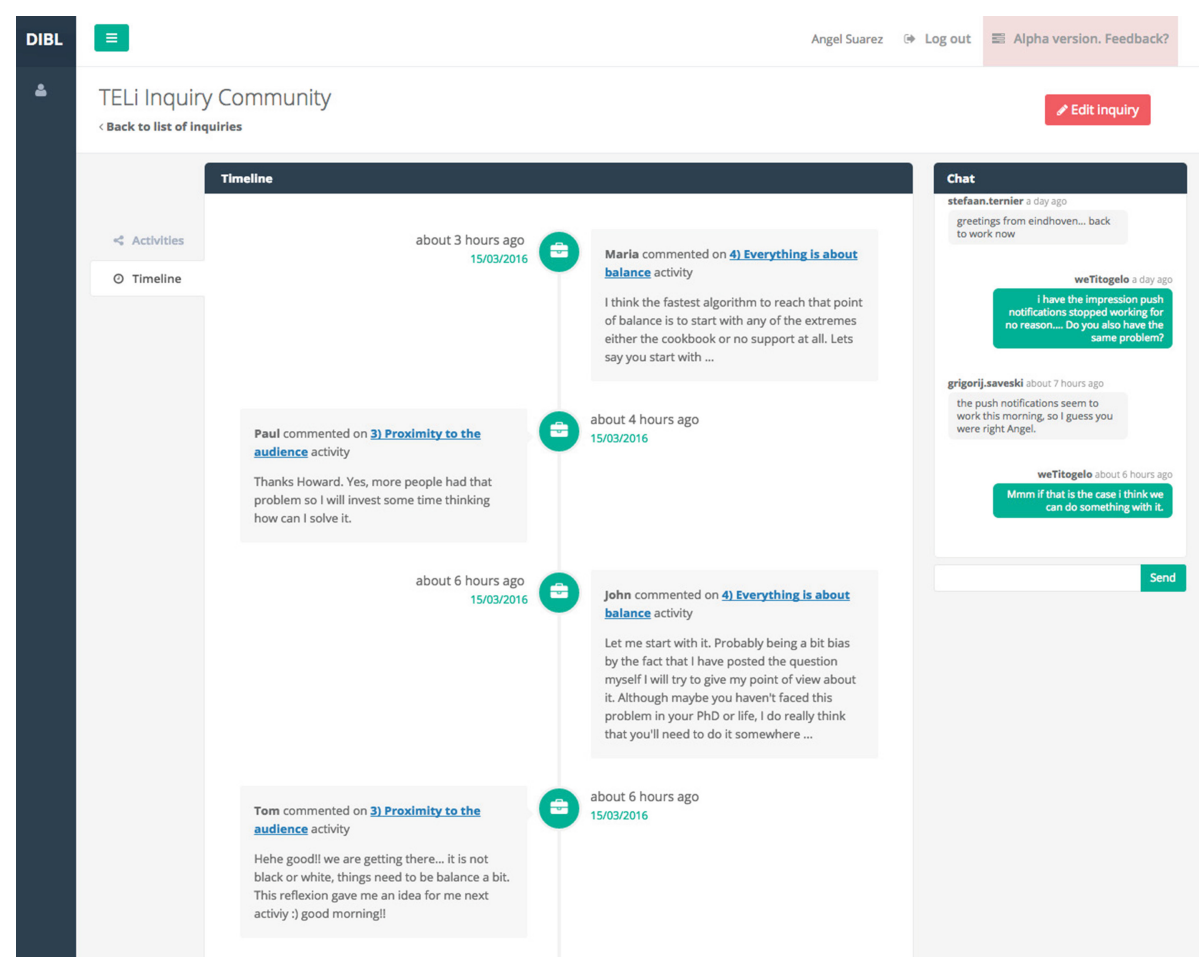

Fig. 3. Inquiry timeline

cognitive, social and teacher presence [11-13]. In addition, using an integrated communication channel external ways of communication are not needed anymore. This avoids the organizational burden of collecting students and teachers phone numbers or accounts to have a shared channel to communicate.

Additionally, DojoIBL implements a notification system and an inquiry timeline as is shown in Fig. 3. The timeline metaphor [35] works as a common ground where teachers and students have a high-level overview of the inquiry progress. Both the timeline and the notification system, promote collaboration awareness based on social, action and activity awareness described in [14]. Many social networks like Facebook ${ }^{\circledR}$ and Twitter ${ }^{\circledR}$ and also educational platforms like ClassDojo and Edmodo provide excellent patterns for communication that are used everyday by a large number of users. Inspired by these patterns, DojoIBL integrates several functionalities to facilitate students' collaboration and communication combined with atomic inquiry elements.

\section{First Formative Study}

DojoIBL will be used in already planned interventions in Dutch schools. In order to address any potential problems with the platform, a formative study was undertaken. The goal of this formative study was to get an understanding of how users perceived the integration of IBL functionalities with social collaborative tools. 
For this experiment we had a total number of 11 experts in the field of Technology Enhanced Learning. Participants were invited to take part in the experiment voluntarily. To get an understanding of how the users perceived DojoIBL, a standardized User Experience Questionnaire (UEQ) [36] was used. The UEQ was designed to obtain a fast and immediate measurement of the user experience of interactive products [37]. It consists of 26 items that measure the perception of a user interface regarding pragmatic, hedonic and attractiveness dimension. Attractiveness represents the overall impression of the product, whereas pragmatic and hedonic are defined as follows.

Pragmatic dimensions include:

- perspicuity: How easy is to get familiar with the product?

- efficiency: Can users solve their tasks without unnecessary effort?

- dependability: Does the user feel control of the interaction?

Hedonic dimensions include:

- stimulation: Is exciting and motivating to use the product?

- novelty: Is the product innovate or creative? Does the product catch the interest of the users?

Attractiveness is represented by 6 items whereas pragmatic and hedonic by four items each. Next to the UEQ, the users perceived usability of DojoIBL was measured using the System Usability Scale (SUS) [38]. SUS is a reliable tool for measuring usability, which consists of 10 items with five possible answers. Both UEQ and SUS are quantitative analysis, therefore to complement the evaluation a semi- structured interview was used. This interview consists of three open questions for collecting more qualitative feedback.

Experimental Design. This formative study lasted for one and a half week. To inform and exhort participants to take part in the experiment, two emails were sent to them. The first one was sent a couple of days before the experiment started and it explained the goal and described the activity. The second email, sent on the same day where the activity started, provided the credentials for the participants to access DojoIBL. Participants were instructed to login DojoIBL, to join one inquiry using an inquiry code and to follow the activities created within the inquiry.

As the goal of the experiment was to know how users perceived the tool, we provided the participants a series of activities based on open ending questions to engage them with DojoIBL. During the time that the activity was running, participants talked in parallel about the topics discussed in DojoIBL. To collect feedback about the user experience (UX) participants were invited to answer questionnaires.

\section{Results}

The 11 participants generated in DojoIBL 260 messages in the chat and 92 responses for the 5 activities created for the inquiry. From those 92 responses, 31 were generated in the concept map and 61 were comments to activities (43 were initial comments and 18 replies to other's comments). The means (ranging from -3 to 3 ) and standard 
deviations (in parenthesis) of the UEQ dimensions for the 11 participants were: attractiveness 2.04 (0.51), perspicuity 1.84 (0.55), efficiency 1.82 (0.51), dependability 1.43 (0.82), stimulation 1.77 (0.61) and novelty 1.61 (0.67). According to these results, participants were equally satisfied with the judgment of hedonic and pragmatic quality dimensions and slightly more satisfied with the attractiveness dimension. For testing the reliability of the dimensions, Conbrach's Alpha was calculated for each dimension. Attractiveness 0.85 , perspicuity 0.7 , dependability 0.69 and stimulation 0.71 showed a satisfactory reliability. Comparing the results to a benchmark based on data from 163 studies, DojoIBL scored in the $10 \%$ best results in all the scales besides dependability.

The overall usability of DojoIBL was rated high by the participants. The mean score for the SUS was 78.0 (12.6). The confidence interval, with confidence level on $95 \%$, ranged from 69.46 to 86.45. For testing reliability Conbrach's Alpha was calculated obtaining 0.81 , which shows a satisfactory reliability. According to what SUS suggests, both the mean and the confidence interval are above 68 which is considered above the average.

From the semi-structured interviews, a number of issues were identified. In five cases, the participants reported problems while navigating back to the phase from the activities. Respondents stressed that going back to the phase overview was not intuitive enough. Also three participants noted problems positioning nodes in the concept maps. The suggestions for improving included a better way to qualify and label the links in the concept map, default inquiry templates while creating new inquiries following existing inquiry models and the integration of learning analytics.

The results, as shown in Fig. 4, confirmed that participants liked DojoIBL and it can be appreciated in several comments like "I really like the social functionality" or "I like the timeline" found in the chat.

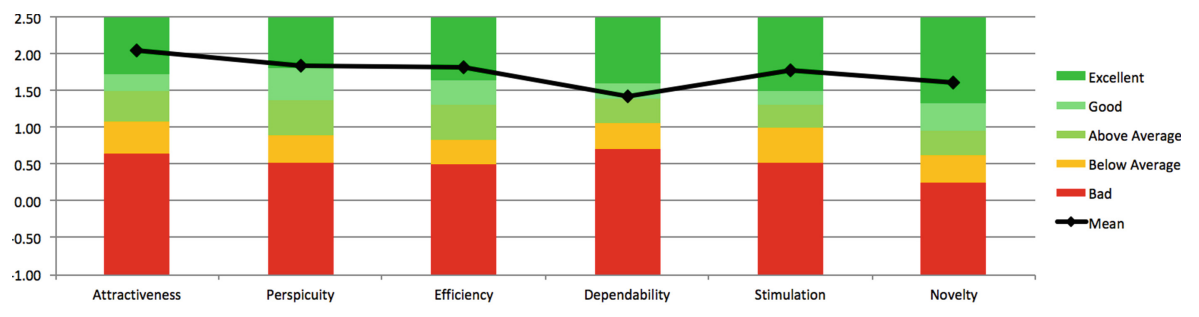

Fig. 4. DojoIBL scores comparison to benchmark

\section{Discussion}

DojoIBL has been developed through a process of design-based research, which promotes progressive refinement of the design [23]. Our conception of social collaborative inquiry learning and its support using DojoIBL motivated the conceptual basis for DojoIBL design, development and refinement leading to the impending interventions in the schools. 
Our goal in this formative study was to gain a better understanding of the way in which the users perceived DojoIBL. In particular, how they perceived the integration of social collaborative tools into an IBL platform. The UEQ scales efficiency, perspicuity and dependability, which measured classical usability, showed that participants perceived DojoIBL as a suitable platform to elaborate and hold discussions around open ended questions. In addition, log data also supported this perception. Participants contributed 8 times on average to activities and they sent on average 23 messages to the chat. The 11 participants were merely instructed to read the description of the activities, having the freedom to contribute or not. Their levels of engagement in social interactions shows that DojoIBL supports social collaborative processes. These interpretations can be confirmed by the SUS questionnaire, where participants, with a high reliability, found the system easy to use and the DojoIBL functionalities very well integrated.

More interpretations can be extracted from the semi-structured interviews. In general participants described the instant messaging as very convenient an intuitive resource to communicate and to ask for specific support. Thus this showed support for two of the components of any educational transaction defined in $\mathrm{CoI}[3,10]$ : social and teaching presence. Regarding cognitive presence, participants found the possibility to discuss around inquiry activities very interesting. They argued that, while instant messaging provides a quick way to communicate an idea, the affordance to also comment on activities provide students time to reflect and to elaborate their contributions. Therefore, this way of communication might be preferable to instant messaging or even oral communication when the goal is to increase high-order cognitive learning [9].

Participants also reflected about the degree of awareness supported. It seemed that social and action awareness [14] were covered with the combination of using notifications and the timeline, as the participants found them convenient to track what others were doing. However, no evidences were reported about the support of activity awareness, which informs users about organizational or structural changes.

In summary, the overall impression from the participants was positive. Besides the feedback that will be addressed and included in the next round of development, participants were excited about the potential of DojoIBL. This was explicitly manifested when some participants showed their interest about future steps of DojoIBL in terms of interventions with students and the roadmap for future updates.

\section{Future Work and Conclusion}

This manuscript presented DojoIBL, a Learning Content Management System that aims at nurturing 'Community of Inquiry' (CoI), by helping students to co-create knowledge through social interactions. It combined essential elements to support inquiry-based learning (IBL) with social collaborative tools in order to facilitate better collaborative processes. In short, DojoIBL focused on adding value to teachers and students' IBL experiences by providing a simple, intuitive and flexible tool.

This formative study informed about how the users perceived DojoIBL, particularly the integration of collaborative tools into an IBL platform. The results showed a 
positive acceptance from participants, perceiving DojoIBL as a suitable tool to engage in collaborative inquiry processes. In addition, the results also showed that DojoIBL copes with the three essential components to any educational transaction described in CoI: cognitive, social and teaching presence.

In future developments of DojoIBL, the integration of role support [39] to enable testing the role taking strategy in IBL processes will be addressed. Roles, as a way to foster communities of inquiry by facilitating interactions between inquirers and fostering positive interdependence [40] will be further investigated. Additionally, although DojoIBL provides a 'liquid design' to be used in any device, a mobile app version is being develop for android, iOS and windows.

To conclude, this manuscript contributed DojoIBL, an open source platform that aims at fostering communities of inquiry for driving students' success facilitating the acquisition of the so called $21^{\text {st }}$ century skills, e.g. communication and collaboration.

Open Access. This chapter is distributed under the terms of the Creative Commons Attribution 4.0 International License (http://creativecommons.org/licenses/by/4.0/), which permits use, duplication, adaptation, distribution and reproduction in any medium or format, as long as you give appropriate credit to the original author(s) and the source, a link is provided to the Creative Commons license and any changes made are indicated.

The images or other third party material in this chapter are included in the work's Creative Commons license, unless indicated otherwise in the credit line; if such material is not included in the work's Creative Commons license and the respective action is not permitted by statutory regulation, users will need to obtain permission from the license holder to duplicate, adapt or reproduce the material.

\section{References}

1. Bruder, R., Prescott, A.: Research evidence on the benefits of IBL. ZDM Math. Educ. 45, 811-822 (2013)

2. Vogel, B., Spikol, D., Kurti, A., Milrad, M.: Integrating mobile, web and sensory technologies to support inquiry-based science learning. In: 6th IEEE International Conference on Wireless, Mobile, and Ubiquitous Technologies in Education, pp. 65-72. IEEE (2010)

3. Peirce, C., Buchler, J.: Philosophical Writings of Peirce. Dover Publications, New York (1955). Selected and Edited, with and Introduction, by Justus Buchler

4. Mikroyannidis, A., Okada, A., Scott, P., Rusman, E., Specht, M., Stefanov, K., Boytchev, P.: weSPOT: a personal and social approach to inquiry-based learning. J. Univ. Comput. Sci. 19(14), 2093-2111 (2013)

5. Bell, T., Urhahne, D.: Collaborative inquiry learning: models, tools, and challenges. Int. J. Sci. Educ. 32(3), 349-377 (2010)

6. Dillenbourg, P.: What do you mean by collaborative learning. Collab. Learn. Cogn. Comput. Approaches 1, 1-15 (1999)

7. Scardamalia, M., Bereiter, C.: Higher levels of agency for children in knowledge building: a challenge for the design of new knowledge media. J. Learn. Sci. 1, 37-68 (1991)

8. Piaget, J.: The Language and Thought of the Child. Harcourt Brace \& Company, New York (1926) 
9. Garrison, D.R., Anderson, T., Archer, W.: Critical inquiry in a text-based environment: computer conferencing in higher education. Internet High. Educ. 2, 87-105 (1999)

10. Pardales, M.J., Girod, M.: Community of inquiry: its past and present future. Educ. Philos. Theor. 38, 299-309 (2006)

11. Garrison, D., Anderson, T., Archer, W.: Critical thinking, cognitive presence, and computer conferencing in distance education. Am. J. Distance Educ. 15, 7-23 (2001)

12. Rourke, L., Anderson, T.: Assessing social presence in asynchronous text-based computer conferencing. Int. J. Distance Educ. 14(3), 51-70 (2007)

13. Anderson, T., Rourke, L., Garrison, D., Archer, W.: Assessing teaching presence in a computer conferencing context. J. Asynchronous Learn. Netw. 5(2), 1-17 (2001)

14. Carroll, J., Rosson, M., Convertino, G., Ganoe, C.: Awareness and teamwork in computer-supported collaborations. Interact. Comput. 18, 21-46 (2006)

15. Carroll, J., Neale, D., Isenhour, P., Rosson, M., McCrickard, D.: Notification and awareness: synchronizing task-oriented collaborative activity. Int. J. Hum. Comput. Stud. 58, 605-632 (2003)

16. Edelson, D.C., Gordin, D.N., Pea, R.D.: Addressing the challenges of inquiry-based learning through technology and curriculum design. J. Learn. Sci. 8, 391-450 (1999)

17. Lehtinen, E.: Computer supported collaborative learning: a review. Reports on Education (1999)

18. van Joolingen, W.R., de Jong, T., Lazonder, A.W., Savelsbergh, E.R., Manlove, S.: Co-Lab: research and development of an online learning environment for collaborative scientific discovery learning. Comput. Hum. Behav. 21, 671-688 (2005)

19. Mulholland, P., Anastopoulou, S., Collins, T., Feisst, M., Gaved, M., Kerawalla, L., Paxton, M., Scanlon, E., Sharples, M., Wright, M.: nQuire: technological support for personal inquiry learning. IEEE Trans. Learn. Technol. 5, 157-169 (2012)

20. Gillet, D., de Jong, T., Sotirou, S., Salzmann, C.: Personalized learning spaces and federated online labs for STEM education at school. In: 2013 IEEE Global Engineering Education Conference (EDUCON), pp. 769-773. IEEE (2013)

21. Bodemer, D., Dehler, J.: Group awareness in CSCL environments. Comput. Hum. Behav. 27(3), 1043-1045 (2011)

22. De Laat, M., Prinsen, F.: Social learning analytics: navigating the changing settings of higher education. Res. Pract. Assess. 9, 51-60 (2014)

23. Barab, S., Squire, K.: Design-based research: putting a stake in the ground. J. Learn. Sci. 13, $1-14(2004)$

24. Specht, M., Bedek, M., Duval, E., Held, P., Okada, A., Stevanov, K., Parodi, E., Kikis-Papadakis, K., Strahovnik, V.: WESPOT: Inquiry based learning meets learning analytics. In: 3rd International Conference on e-Learning, pp.15-20. Belgrade, Serbia (2012)

25. Pedaste, M., Mäeots, M., Siiman, L.A., de Jong, T., van Riesen, S.A.N., Kamp, E.T., Manoli, C.C., Zacharia, Z.C., Tsourlidaki, E.: Phases of inquiry-based learning: definitions and the inquiry cycle. Educ. Res. Rev. 14, 47-61 (2015)

26. Donohoo, J.: Collaborative Inquiry for Educators: A Facilitator's Guide to School Improvement. HarperCollins, New York (2013)

27. Dillenbourg, P., Järvelä, S., Fischer, F.: The evolution of research on computer-supported collaborative learning. In: Balacheff, N., Ludvigsen, S., de Jong, T., Lazonder, A., Barnes, S. (eds.) Technology-Enhanced Learning, pp. 3-19. Springer, Netherlands (2009)

28. Hmelo-silver, C.E., Duncan, R.G., Chinn, C.A.: Scaffolding and achievement in problem-based and inquiry learning: a response to Kirschner, Sweller, and Clark (2006). Educ. Psychol. 42, 99-107 (2007) 
29. Ternier, S., Klemke, R., Kalz, M., Specht, M.: ARLearn: augmented reality meets augmented virtuality. J. Univ. Comput. Sci. Technol. Learn Across Phys. Virtual Spaces 18 (15), 2143-2164 (2012)

30. Tafoya, E., Sunal, D.W., Knecht, P.: Assessing inquiry potential: a tool for curriculum decision makers. Sch. Sci. Math. 80, 43-48 (1980)

31. Ahern-Rindell, A.: Applying inquiry-based and cooperative group learning strategies to promote critical thinking. J. Coll. Sci. Teach. 28(3), 203-207 (1998)

32. Stoddart, T., Abrams, R.: Concept maps as assessment in science inquiry learning - a report of methodology. J. Sci. Educ. 22(12), 1221-1246 (2000)

33. Akinsanya, C., Williams, M.: Concept mapping for meaningful learning. Nurse Educ. Today 24(1), 41-46 (2004)

34. Garrison, D.: Critical thinking and self-directed learning in adult education: an analysis of responsibility and control issues. Adult Educ. Q. 36, 60-64 (1992)

35. Vavoula, G., Sharples, M.: KLeOS: a personal, mobile, knowledge and learning organisation system. In: IEEE International Workshop on Wireless and Mobile Technologies in Education (2002)

36. Laugwitz, B., Held, T., Schrepp, M.: Construction and evaluation of a user experience questionnaire. In: Holzinger, A. (ed.) USAB 2008. LNCS, vol. 5298, pp. 63-76. Springer, Heidelberg (2008)

37. Santoso, H., Barat, I.J., Schrepp, M.: Measuring user experience of the student-centered e-Learning environment. thejeo.com

38. Sauro, J.: Measuring usability with the system usability scale (SUS) (2011)

39. Strijbos, J.-W., De Laat, M.F.: Developing the role concept for computer-supported collaborative learning: an explorative synthesis. Comput. Hum. Behav. 26, 495-505 (2010)

40. Johnson, D., Johnson, R., Smith, K.: Active Learning: Cooperation in the College Classroom. Interaction Book Company, Edina (1991) 